\title{
SUSY after LHC run 1
}

\section{Heidi Rzehak*}

Albert-Ludwigs-Universität Freiburg, Physikalisches Institut, Hermann-Herder-Str. 3, 79104

Freiburg, Germany

E-mail: heidi.rzehakephysik.uni-freiburg.de

The first run of the LHC has been a great success. Not only a Higgs boson has been discovered but also many searches for physics beyond the Standard Model have been performed. In particular, supersymmetric partner particles have been searched for. Both, the discovery of a Higgs boson and the searches, constrain models with an underlying supersymmetry. Some of the resulting consequences for viable supersymmetric models will be discussed.

Frontiers of Fundamental Physics 14 - FFP14,

15-18 July 2014

Aix Marseille University (AMU) Saint-Charles Campus, Marseille

${ }^{*}$ Speaker. 


\section{Introduction}

Models with an underlying supersymmetry (SUSY), a symmetry which relates fermionic and bosonic degrees of freedom, are among the most intensively studied models going beyond the Standard Model. Theoretically appealing is the fact that supersymmetry is the only symmetry combining internal and space-time symmetries in a non-trivial way [1, 2].

The minimal supersymmetric extension of the Standard Model (SM), the Minimal Supersymmetric Standard Model (MSSM), comprises the Standard Model particles and an additional Higgs doublet which is needed to generate the masses of up- and down-type fermions as well as to keep the theory anomaly-free, i.e. the MSSM is based on a Two-Higgs-Doublet model (2HDM) (of type II). In addition, fields are added in such a way that each fermionic 2HDM-type degree of freedom possesses a bosonic superpartner one and vice versa. This greatly enlarges the particle content with respect to the SM. One of the features of exact SUSY is that the mass of the 2HDM-type particle and the one of its superpartner are the same, which is experimentally excluded since no superpartners have been discovered so far. In the MSSM, SUSY is broken explicitly by adding so-called soft-SUSY breaking terms, which do not change the relations between dimensionless couplings. Via the soft-SUSY breaking part, many parameters, where some of them are possibly complex, are introduced. Often, an additional symmetry is imposed, the discrete R-parity. As a consequence of the R-parity, the lightest superpartner particle (LSP) is stable.

There are several reasons why supersymmetric extensions of the Standard Model are considered to solve some of the problems of the Standard Model. For example, experimental observations suggest the existence of dark matter, which cannot be explained solely within the Standard Model. An R-parity conserving supersymmetric model, however, exhibits a dark matter candidate in terms of the LSP [3]. Furthermore, there are also open questions from the theory side which can be answered within supersymmetric models. For instance, grand unification can be realized in these models while the Standard Model does not offer this possibility [4]. Also, the so-called hierarchy problem can be addressed by supersymmetric models [5]: The squared mass of the Higgs boson in the Standard Model depends quadratically on the scale up to which the Standard Model is valid. If this scale was the Planck scale, the scale at which effects of gravity should become non-negligible, large corrections to the bare mass would be introduced; in order to obtain a Higgs mass at the observed value, large cancellations would be necessary. In supersymmetric models, the underlying supersymmetry protects the Higgs mass squared from these large contributions.

The first summary of the results of the first run of the LHC with respect to supersymmetric models is: A Higgs boson of mass of $125 \mathrm{GeV}$ has been discovered [6, 7]. This newly discovered particle could be one of the predicted Higgs bosons in supersymmetric models. Additionally, the allowed parameter space of supersymmetric models has been constrained by direct searches for superpartners and further Higgs bosons as well as by indirect measurements e.g. of the mass of the discovered Higgs boson. In the following, we will consider some details of these constraints.

\section{Constrained MSSM}

As mentioned in the introduction, via the soft-SUSY breaking terms, many new parameters, of order $\mathscr{O}(100)$, have been introduced into the model. In order to perform an analysis, it is desirable 
to reduce the number of free parameters. One way of reducing the number of parameters is, instead of considering the most general model, to apply additional assumptions and constrain the number of free parameters. The constrained MSSM (CMSSM) is such a model [8]. It is inspired by gravitymediated SUSY-breaking and reduces the number of new parameters to 4 parameters and one sign. The parameters are usually given at the grand unification (GUT) scale. Via the renormalization group equations (RGE), the parameters are evolved down to the phenomenologically relevant scale, often $1 \mathrm{TeV}$. The parameters are the scalar soft-breaking mass parameter $m_{0}$, the gaugino mass parameter $m_{\frac{1}{2}}$, the scalar trilinear couplings $A_{0}$, the ratio of the vacuum expectation values $\tan \beta=$ $\frac{v_{d}}{v_{u}}$, and the sign of the Higgs superfield mixing parameter, $\operatorname{sign}(\mu)$.

The first results of LHC run 1 [9], which excluded parts of the SUSY parameter space, were based on the CMSSM. The main exclusion came from the measurement of events with many jets and missing transverse energy. The exclusion bounds shown by ATLAS at ICHEP2014 also include processes with several leptons such as processes with three charged leptons or two leptons with the same charge [10]. Still, processes with many jets, also with many b-jets result in the strongest constraints. Several groups have studied the CMSSM, including the experimental exclusion bounds and indirect constraints such as the Higgs boson mass, see e.g. Refs. [11, 12]. The resulting SUSY spectra contains rather heavy supersymmetric partner particle, with squarks of the first two generations of around $2 \mathrm{TeV}$, a light supersymmetric partner of the top quark (stop) of $\sim 750 \mathrm{GeV}$ and a neutralino LSP of around $500 \mathrm{GeV}$ [11]. Additionally, a tension has been observed between the $g-2$ results, since, for an improvement of the agreement of the theoretical prediction of $g-2$ and the experimental measurements compared to the Standard Model, rather light smuons are needed.

\subsection{The mass of the Higgs boson}

For the application of indirect constraints, not only the experimental value of the considered observable, but also a precise theoretical prediction of its value is needed. Since the limits of the direct searches of superpartners point to rather heavy superpartners, in particular in constrained models like the CMSSM, a good theoretical prediction for large SUSY mass scales $M_{S}$ is also necessary. Fixed-order calculations of the Higgs mass contain logarithms of the form $\log \left(\mathrm{M}_{\mathrm{S}} / \mathrm{m}_{\mathrm{t}}\right)$ which can become large and spoil the accuracy of the prediction. For large mass scales, a better approach is the calculation of the Higgs mass with the help of RGE. Applying this approach, the large logarithms are resummed and the prediction for large SUSY mass scales is improved (for the latest Higgs mass result using the RGE approach, see Ref. [13]). In order to be able to scan from smaller scales to larger scales, a combination of the fixed-order and the RGE approach can be useful. A first combination of both approaches [15] has been implemented into the program FeynHiggs [14, 15], leading to an improved Higgs mass prediction for SUSY mass scales larger than $\sim 1 \mathrm{TeV}$ where the logarithms of higher orders become important. In Fig. 1, a fit of the CMSSM taking into account indirect and direct constraints is shown [16]. The old Higgs mass values in the $m_{0}-m_{\frac{1}{2}}$ plane are shown in red dashed lines while the improved Higgs mass result is presented as solid black lines. The blue region adjacent to the brown region, where the LSP is a charged particle, is the region with the right relic density. Using the old Higgs mass prediction, one would conclude that this blue region is not compatible with the measured Higgs mass value, while with the improved prediction it is. For these analyses, it is important to have precise predictions with a good error estimate. 


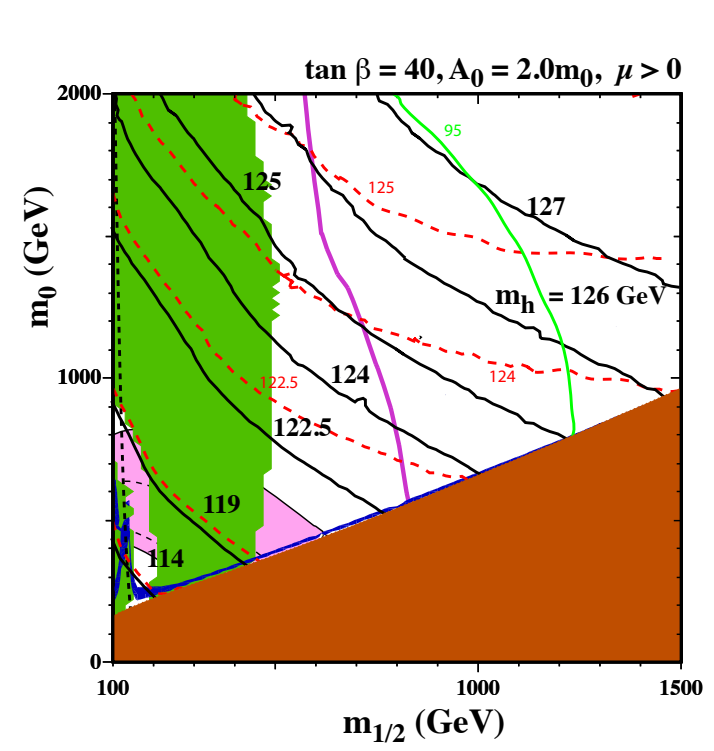

Figure 1: The $m_{0}-m_{\frac{1}{2}}$ plane with different constraints is shown: The black solid line is the Higgs mass result obtained with FeynHiggs 2.10 .0 [14, 15], while the red dashed curve displays the one obtained with FeynHiggs2.8.6 [14]. The green line depicts the $95 \% \mathrm{CL}$ limit of $\mathrm{BR}\left(B_{s} \rightarrow \mu \mu\right)$ measured by CMS and LHCb [17]. The 95\% CL exclusion limit established by ATLAS following searches for missing transverse energy events using $20 \mathrm{fb}^{-1}$ of the $\mathrm{LHC}$ data at $8 \mathrm{TeV}$ is presented as a violet line [18]. The blue strips depict regions where the relic density is $0.06<\Omega_{\chi} h^{2}<0.2$. This less restrictive requirement is chosen for better visibility otherwise the bound $0.115<\Omega_{\chi} h^{2}<0.125$ [19] has been applied in Ref. [16]. The magenta region presents the parameter space favoured by the SUSY interpretation of $g-2$ [20]. The green shaded region depicts the $\mathrm{BR}(b \rightarrow s \gamma)$ exclusion limit at the $2-\sigma$ level. In the brown region the LSP is charged. This figure is taken from Ref. [16].

\section{Beyond the CMSSM}

Taking into account direct and indirect measurements, the CMSSM, though not excluded, is very strongly constrained. It might very well be that this model oversimplifies the situation realised in nature. Now, there are two possibilities to proceed: First, starting from the CMSSM, one can relax the assumptions and consider slightly more general models like the Non-UniversalHiggs-Mass model [21], where the soft-SUSY-breaking parameters of the Higgs sector are treated independently of the soft-SUSY-breaking parameters of the sfermions, or the so called phenomenological MSSM (pMSSM) where simplifying relations between some of the parameters are assumed. Usually, the number of considered free parameters is specified in the name, e.g. pMSSM8. Second, another approach is to try to capture special properties of the considered SUSY particles and to construct a simplified model [22]. These simplified models, however, are not embedded in a complete theoretical model.

\subsection{Bounds on masses of first generation of squarks}

In one of the simplified models, the decay of a squark into a quark and neutralino, $\tilde{q} \rightarrow q \chi_{1}^{0}$, with a branching ratio of $100 \%$ is considered. The gluino is assumed to be decoupled. It is important to note that the experimental analyses assumed degenerate squark masses for the first two generations, yielding 8 squarks with the same mass, see e.g. Ref. [23]. In a model, where only one of these squarks is light, the bounds are less strict and a single squark of mass of $\mathscr{O}(400 \mathrm{GeV})$ is still allowed according to the $7 \mathrm{TeV}$ data, while the degenerate squarks are excluded up to masses of $\mathscr{O}(800 \mathrm{GeV})$ [24]. For the interpretation, it is very important to consider the details of the assumptions that went into the considered simplified model.

\subsection{Scalar top quarks}

The coupling of the stops to the Higgs boson is related to the one of the top quarks to the 
Higgs boson. The size of both Higgs couplings, the one of the top quarks and the one of their superpartners, are relatively large. This connection to the Higgs sector makes the stops particularly interesting. The size of the stop masses and mixings plays an important role in the prediction of the mass of the Higgs boson and, hence, in the discussion of how much fine-tuned a particular supersymmetric model is, see e.g. Ref. [25].

The mixing of the squarks is proportional to the mass of the quark partner. Thus, in the case of the stops, it is proportional to the top quark mass and can be rather large while in the first two generations the mixing is suppressed by the smallness of the corresponding quark masses.

Dedicated searches for stops have been performed, and, in specific scenarios, stops with masses smaller than $750 \mathrm{GeV}$ are excluded [26]. The searches are performed exploiting simplified models with specific decay chains of the stops. Again, it is very important to take into account the particular assumptions when deciding whether a certain parameter point is excluded or not. For some stop mass ranges, the searches are especially difficult. In the simplified model, in which it is assumed that a stop decays into a top quark and a neutralino with $100 \%$ branching ratio, the separation of the top quark background from a possible signal is particularly difficult in the region where the stop and the top quark have roughly equal masses. In Ref. [27], this part of parameter space is tackled by considering the top pair production cross sections. Instead of exploiting differences of the production and decay mode, in this case, the similarities of the decay properties of the top quark and the stops result in a simultaneous inclusive measurement of top and stop cross section, such that, compared to the Standard Model, one should observe a larger cross section if a stop in this mass range exists. Another complementary way to get a handle on stops in this mass range are investigations of spin correlations in top pair production with subsequent decay into a bottom quark and a leptonically decaying $\mathrm{W}$. The best sensitivity can be obtained measuring the azimuthal angle difference of the leptons in the low invariant $t \bar{t}$ mass region [28]. An excess of uncorrelated events could be due to stops [29].

\subsection{Electroweakly interacting supersymmetric particles}

Searches for neutralinos and charginos are more difficult than for first generations of squarks, since they are only electroweakly interacting and no jets are involved. The best limits come from trilepton searches where the Standard Model background is low, see e.g. Ref. [30].

\subsection{Deviations from Standard Model cross sections}

Complementary to direct searches, measured deviations from theoretically predicted values of Standard Model observables can give hints for new physics. A slight upward deviation of the measured $W W$ cross section compared to the Standard Model expectation has been observed [31], up to a $2-2.5 \sigma$ level. This deviation could be explained by the existence of supersymmetric partner particles which contaminate the measurement. Scenarios with charginos, sleptons and stops have been discussed [32]. Also, the contamination of the $W W$ background to Higgs searches by new physics particles and resulting errors of the measured Higgs couplings have been addressed [33]. However, it is not clear whether this deviation results from new physics. One explanation of this upward deviation within the Standard Model: For the conversion from the fiducial cross section to the total cross section an extrapolation has to be done and an error might be introduced by the used Monte-Carlo prediction [34]. 


\section{Conclusion}

During the first run of the LHC, a Higgs boson has been discovered and the SUSY parameter space has been constrained significantly by searches of jets, $b$-jets, missing energy and no or one lepton, by trilepton searches, and dedicated searches of specific characteristic scenarios. However, there remains allowed unconstrained parameter space and an exciting LHC run 2 is ahead of us.

\section{Acknowledgments}

H.R. would like to thank the organizers of "Frontiers of Fundamental Physics 2014" for the kind invitation to give this talk and for an interesting and enjoyable conference.

\section{References}

[1] S. Coleman, J. Mandula, Phys. Rev. 159 (1967) 1251.

[2] R. Haag, J.T. Łopuszánski, M. Sohnius, Nucl. Phys. B 88 (1975) 257.

[3] H. Goldberg, Phys. Rev. Lett. 50 (1983) 1419 [Erratum-ibid. 103 (2009) 099905], J. R. Ellis, J. S. Hagelin, D. V. Nanopoulos, K. A. Olive, M. Srednicki, Nucl. Phys. B 238 (1984) 453.

[4] J. R. Ellis, S. Kelley, D. V. Nanopoulos, Phys. Lett. B 260 (1991) 131, U. Amaldi, W. de Boer, H. Furstenau, Phys. Lett. B 260 (1991) 447, P. Langacker, M. x. Luo, Phys. Rev. D 44 (1991) 817. C. Giunti, C. W. Kim, U. W. Lee, Mod. Phys. Lett. A 6 (1991) 1745.

[5] E. Witten, Nucl. Phys. B 188 (1981) 513.

[6] G. Aad et al. [ATLAS Collaboration], Phys. Lett. B 716 (2012) 1.

[7] S. Chatrchyan et al. [CMS Collaboration], Phys. Lett. B 716 (2012) 30.

[8] see e.g. G. L. Kane, C. F. Kolda, L. Roszkowski, J. D. Wells, Phys. Rev. D 49 (1994) 6173.

[9] V. Khachatryan et al. [CMS Collaboration], Phys. Lett. B 698 (2011) 196; G. Aad et al. [ATLAS Collaboration], Phys. Rev. Lett. 106 (2011) 131802.

[10] G. Aad et al. [ATLAS Collaboration], JHEP 1409 (2014) 176; ATLAS Collaboration, https: / / atlas.web.cern. ch/Atlas/GROUP S / PHYS ICS / CombinedSummaryPlots / SUSY/ATLAS_SUSY_MSUGRA/ATLAS_SUSY_MSUGRA.pdf.

[11] P. Bechtle, T. Bringmann, K. Desch, H. Dreiner, M. Hamer, C. Hensel, M. Krämer, N. Nguyen et al., JHEP 1206 (2012) 098; P. Bechtle, K. Desch, H. K. Dreiner, M. Hamer, M. Krämer, B. O’Leary, W. Porod, X. Prudent et al., PoS EPS -HEP2013 (2013) 313.

[12] K. Kowalska, L. Roszkowski, E. M. Sessolo, JHEP 1306 (2013) 078; O. Buchmueller, R. Cavanaugh, A. De Roeck, M. J. Dolan, J. R. Ellis, H. Flacher, S. Heinemeyer, G. Isidori et al., Eur. Phys. J. C 74 (2014) 6, 2922.

[13] P. Draper, G. Lee, C. E. M. Wagner, Phys. Rev. D 89 (2014) 5, 055023.

[14] S. Heinemeyer, W. Hollik, G. Weiglein, Comput. Phys. Commun. 124 (2000) 76; Eur. Phys. J. C 9 (1999) 343; G. Degrassi, S. Heinemeyer, W. Hollik, P. Slavich, G. Weiglein, Eur. Phys. J. C 28 (2003) 133; M. Frank, T. Hahn, S. Heinemeyer, W. Hollik, H. Rzehak, G. Weiglein, JHEP 0702 (2007) 047.

[15] T. Hahn, S. Heinemeyer, W. Hollik, H. Rzehak, G. Weiglein, Phys. Rev. Lett. 112 (2014) 14, 141801. 
[16] O. Buchmueller, M. J. Dolan, J. Ellis, T. Hahn, S. Heinemeyer, W. Hollik, J. Marrouche, K. A. Olive et al., Eur. Phys. J. C 74 (2014) 3, 2809.

[17] S. Chatrchyan et al. [CMS Collaboration], Phys. Rev. Lett. 111 (2013) 101804; R.Aaij et al. [LHCb Collaboration], Phys. Rev. Lett. 111 (2013) 101805 ; R.Aaij et al. [LHCb and CMS Collaborations], LHCb-CONF-2013-012, CMS PAS BPH-13-007 (2013).

[18] ATLAS Collaboration, https://twiki.cern.ch/twiki/bin/view/AtlasPublic/ CombinedSummaryP lots\# SusyMSUGRASummary.

[19] P. A. R. Ade et al. [Planck Collaboration], arXiv:1303.5076 [astro-ph.CO].

[20] T. Blum, A. Denig, I. Logashenko, E. de Rafael, B. L. Roberts, T. Teubner, G. Venanzoni, arXiv:1311.2198 [hep-ph].

[21] A. Lleyda, C. Munoz, Phys. Lett. B 317 (1993) 82; M. Olechowski, S. Pokorski, Phys. Lett. B 344 (1995) 201; V. Berezinsky, A. Bottino, J. R. Ellis, N. Fornengo, G. Mignola, S. Scopel, Astropart. Phys. 5 (1996) 1.

[22] J. Alwall, P. Schuster, N. Toro, Phys. Rev. D 79 (2009) 075020.

[23] G. Aad et al. [ATLAS Collaboration], Phys. Rev. D 87 (2013) 1, 012008; S. Chatrchyan et al. [CMS Collaboration], JHEP 1301 (2013) 077.

[24] R. Mahbubani, M. Papucci, G. Perez, J. T. Ruderman, A. Weiler, Phys. Rev. Lett. 110 (2013) 15, 151804.

[25] L. J. Hall, D. Pinner, J. T. Ruderman, JHEP 1204 (2012) 131.

[26] G. Aad et al. [ATLAS Collaboration], JHEP 1409 (2014) 015; JHEP 1411 (2014) 118; JHEP 1406 (2014) 124; S. Chatrchyan et al. [CMS Collaboration], Eur. Phys. J. C 73 (2013) 12, 2677; CMS Collaboration [CMS Collaboration], CMS-PAS-SUS-14-011.

[27] M. Czakon, A. Mitov, M. Papucci, J. T. Ruderman, A. Weiler, Phys. Rev. Lett. 113 (2014) 20, 201803.

[28] G. Mahlon, S. J. Parke, Phys. Rev. D 81 (2010) 074024.

[29] Z. Han, A. Katz, arXiv:1310.0356 [hep-ph]; G. Aad et al. [ATLAS Collaboration], arXiv:1412.4742 [hep-ex].

[30] G. Aad et al. [ATLAS Collaboration], JHEP 1404 (2014) 169; JHEP 1405 (2014) 071 ; V. Khachatryan et al. [CMS Collaboration], Eur. Phys. J. C 74 (2014) 9, 3036; Phys. Rev. D 90 (2014) 9, 092007.

[31] G. Aad et al. [ATLAS Collaboration], Phys. Rev. D 87 (2013) 11, 112001 [Erratum-ibid. D 88 (2013) 7, 079906]; The ATLAS collaboration, ATLAS-CONF-2014-033; S. Chatrchyan et al. [CMS Collaboration], Phys. Lett. B 721 (2013) 190; Eur. Phys. J. C 73 (2013) 10, 2610.

[32] D. Curtin, P. Jaiswal, P. Meade, Phys. Rev. D 87 (2013) 3, 031701; D. Curtin, P. Jaiswal, P. Meade, P. J. Tien, JHEP 1308 (2013) 068; K. Rolbiecki, K. Sakurai, JHEP 1309 (2013) 004; D. Curtin, P. Meade, P. J. Tien, Phys. Rev. D 90 (2014) 11, 115012; J. S. Kim, K. Rolbiecki, K. Sakurai, J. Tattersall, JHEP 1412 (2014) 010.

[33] B. Feigl, H. Rzehak, D. Zeppenfeld, Phys. Lett. B 717 (2012) 390.

[34] P. F. Monni, G. Zanderighi, arXiv:1410.4745 [hep-ph]. 